\title{
Article
}

\section{Social Competence in Parents Increases Children's Educational Attainment: Replicable Genetically-Mediated Effects of Parenting Revealed by Non-Transmitted DNA}

\author{
Timothy C. Bates ${ }^{1}$, Brion S. Maher ${ }^{2}$, Lucía Colodro-Conde 3 , Sarah E. Medland ${ }^{3}$, Kerrie McAloney ${ }^{3}$, \\ Margaret J. Wright ${ }^{4,5}$, Narelle K. Hansell ${ }^{4}$, Aysu Okbay ${ }^{6}$, Kenneth S. Kendler ${ }^{7}$, Nicholas G. Martin ${ }^{3}$ and \\ Nathan A. Gillespie ${ }^{7}$ \\ ${ }^{1}$ Department of Psychology, University of Edinburgh, Edinburgh, UK, ${ }^{2}$ Johns Hopkins Bloomberg School of Public Health, Baltimore, MD, USA, \\ ${ }^{3}$ QIMR Berghofer Medical Research Institute, Brisbane, Queensland, Australia, ${ }^{4}$ Queensland Brain Institute, University of Queensland, Brisbane, \\ Queensland, Australia, ${ }^{5}$ Centre for Advanced Imaging, University of Queensland, Brisbane, Queensland, Australia, ${ }^{6}$ Department of Economics, \\ School of Business and Economics, Vrije Universiteit Amsterdam, Amsterdam, The Netherlands and ${ }^{7}$ Virginia Institute of Psychiatric and \\ Behavioral Genetics, Virginia Commonwealth University, Richmond, VA, USA
}

\begin{abstract}
We recently reported an association of offspring educational attainment with polygenic risk scores (PRS) computed on parent's nontransmitted alleles for educational attainment using the second GWAS meta-analysis article on educational attainment published by the Social Science Genetic Association Consortium. Here we test the replication of these findings using a more powerful PRS from the third GWAS meta-analysis article by the Consortium. Each of the key findings of our previous paper is replicated using this improved PRS $(N=2335$ adolescent twins and their genotyped parents). The association of children's attainment with their own PRS increased substantially with the standardized effect size, moving from $\beta=0.134,95 \% \mathrm{CI}=0.079,0.188$ for $\mathrm{EA} 2$, to $\beta=0.223,95 \% \mathrm{CI}=0.169,0.278, p<.001$, for $\mathrm{EA} 3$. Parent's PRS again predicted the socioeconomic status (SES) they provided to their offspring and increased from $\beta=0.201,95 \% \mathrm{CI}=0.147$, 0.256 to $\beta=0.286,95 \% \mathrm{CI}=0.239,0.333$. Importantly, the PRS for alleles not transmitted to their offspring — therefore acting via the parenting environment - was increased in effect size from $\beta=0.058,95 \% \mathrm{CI}=0.003,0.114$ to $\beta=0.067,95 \% \mathrm{CI}=0.012,0.122, p=.016$. As previously found, this non-transmitted genetic effect was fully accounted for by parental SES. The findings reinforce the conclusion that genetic effects of parenting are substantial, explain approximately one-third the magnitude of an individual's own genetic inheritance and are mediated by parental socioeconomic competence.
\end{abstract}

Keywords: educational attainment; parenting; parental environment; non-transmitted genotype; virtual-parent design; polygenic risk scores; PRS; socioeconomic status; SES

(Received 6 December 2018; accepted 13 December 2018)

We recently reported an association of educational attainment with polygenic risk scores (PRS) computed using the $50 \%$ of DNA not transmitted to offspring by their parents (Bates et al., 2018), a finding also demonstrated elsewhere (Koellinger \& Harden, 2018; Kong et al., 2018). Those findings were based on the second GWAS meta-analysis on educational attainment published by the Social Science Genetic Association Consortium (EA2; Okbay et al., 2016). Here, we use the pseudo-control method (Cordell et al., 2004) and the recently available third phase of educational attainment scores (EA3; Lee et al., 2018) to replicate this finding with a stronger polygenic predictor. We employed the same sample and high-quality measure of educational attainment, testing the replication of the hypotheses that: offspring EA3 PRS would positively predict their educational attainment $(\mathrm{H} 1)$; parents' EA3 PRS would positively predict their attained SES (H2); and offspring's educational attainment would be significantly associated with parental EA3 PRS computed from alleles not transmitted to their offspring (H3). Finally, this cultural transmission effect would operate via socioeconomic competence at creating the extended phenotype of environmental niches aiding offspring development (H4).

\section{Methods}

Author for correspondence: Timothy C. Bates, Email: tim.bates@ed.ac.uk Cite this article: Bates TC, Maher BS, Colodro-Conde L, Medland SE, McAloney K, Wright MJ, Hansell NK, Okbay A, Kendler KS, Martin NG, and Gillespie NA. (2019) Social Competence in Parents Increases Children's Educational Attainment: Replicable Genetically-Mediated Effects of Parenting Revealed by Non-Transmitted DNA. Twin Research and Human Genetics 22: 1-3, https://doi.org/10.1017/thg.2018.75

\section{Subjects}

The sample consisted of all 2335 adolescent twins and their genotyped parents available within the Brisbane Adolescent Twin Study (Wright \& Martin, 2004) for whom educational attainment

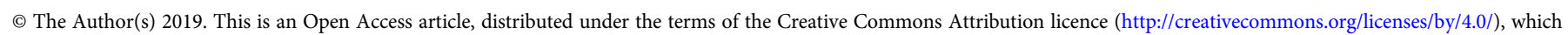
permits unrestricted re-use, distribution, and reproduction in any medium, provided the original work is properly cited. 
Table 1. Comparison of effect sizes between EA2 and EA3 polygenic risk scores as predictors of offspring educational attainment and parental SES

\begin{tabular}{lcr}
\hline Hypothesis & EA2 $\beta$ and 95\% confidence interval & EA3 $\beta$ and 95\% confidence interval \\
\hline PRS effect on attainment & $0.133[0.078,0.187]$ & $0.223[0.169,0.277]$ \\
\hline PRS effect on parent SES & $0.201[0.147,0.256]$ & $0.286[0.239,0.333]$ \\
\hline Non-transmitted PRS on attainment & $0.058[0.003,0.114]$ & $0.068[0.014,0.123]$ \\
\hline
\end{tabular}

Fig. 1. Prediction of offspring educational attainment from offspring EA3 polygenic risk scores (A) and by parental non-transmitted EA3 polygenic risk scores (B).
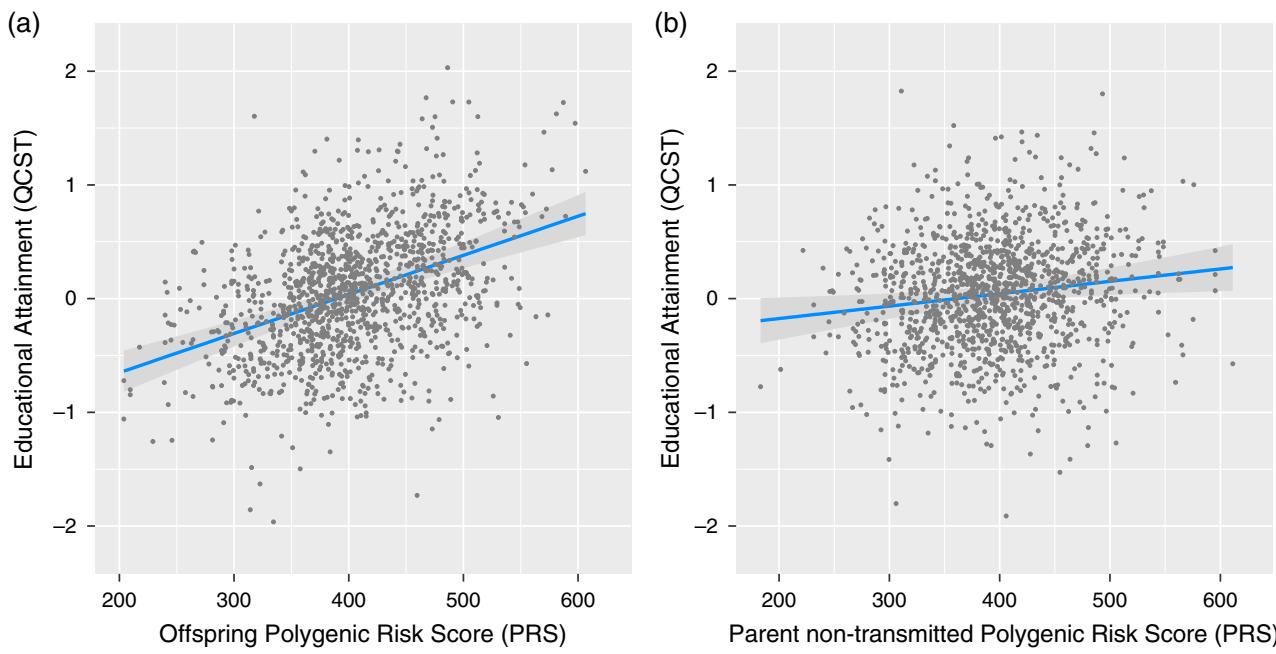

data and genotype data are available (1333 female, mean age at testing 17.15 years, $S D=0.39 ; 1002$ male, mean age at testing 17.2 years, $S D=0.41$ ), along with genotype and SES data for their parents. Exclusion criteria for entry to the cohort were significant head injury, neurological or psychiatric illness, substance dependence or chronic use of medications with central nervous system effects. Subjects were genotyped on the $610 \mathrm{~K}$ and GSA Illumina genome-wide SNP platform and imputed to the Haplotype Reference Consortium (version 1.1). The study was approved by the QIMR Berghofer Medical Research Institute's Human Research Ethics Committee.

\section{Phenotypic Measures}

Educational attainment was assessed using the overall score for the Queensland Core Skills Test (QCST; Queensland Studies Authority, 2003): a 7-h comprehensive assessment completed in the final year of high school (age $\sim 17$ years) and providing a global index of achievement, standardized to the state-wide sample means for each year. Childhood SES was assessed using the Australian Socioeconomic Index 2006 (AUSEI06) occupational status scale (McMillan et al., 2009) and was ascertained for each parent $~ 12$ months prior to the twins taking the QCST. For each twin pair, parental SES was set to the maximum of their maternal and paternal values.

\section{Genetic Measures}

Non-transmitted parental genomes were created using PseudoCons (Cordell et al., 2004; Howey, 2014). Polygenic risk scores for EA3 were calculated for the offspring and parental pseudocontrol genomes (best-guess imputed genotypes, excluding SNPs with low imputation quality $r^{2}<.6$ and MAF $<1 \%$ ) using PLINK1.9 (Purcell et al., 2007) and the method described by Wray et al. (2014). A standardized PRS was computed using
79,339 SNPs: all SNPs associated $p \leq .05$ recomputed from the cohorts of the third educational attainment GWAS (Lee et al., 2018) leaving out the QIMR and 23andMe samples, and using European population LD information from the 1000 Genomes reference set (pruning criteria LD $r^{2}<.1$ within windows of $10 \mathrm{Mb}$ ).

Multilevel modeling was completed using lme from the nlme package (Pinheiro et al., 2017) to construct multilevel models with QCST as the dependent variable, with predictors nested within family and zygosity. Analyses controlled for age at test and for sex.

\section{Results}

We tested the replication of the four hypotheses outlined above. Results for hypotheses 1-3 are tabulated in Table 1 for convenience. Replicating hypothesis 1 , educational attainment in the offspring was significantly related to their own EA3 PRS in a multi-level model with offspring QCST as the dependent variable, with offspring EA3 PRS, age and sex as predictors $(\beta=0.223,95 \%$ $\mathrm{CI}=0.169,0.277, t(606)=8.045, p<.001$; see Figure 1 , panel A). This exceeds the beta found using EA2 PRS $(\beta=0.133 ; 95 \%$ $\mathrm{CI}=0.078,0.187, t(606)=4.805, p<.001)$. Controlling for parental SES did not materially alter this effect. Updating hypothesis 2, a linear model predicting parental attained SES from parental EA3 PRS was significant $(\beta=0.29,95 \% \mathrm{CI}=0.24,0.33, t=11.98$, $p<.001)$. This was again larger than was found using EA2 $(\beta=0.20$, $95 \% \mathrm{CI}=0.15,0.26)$. Regarding an ancillary hypothesis that subjects' EA3 PRS would interact with their parents' SES (TuckerDrob \& Bates, 2016) - that is, a $\mathrm{G}_{\mathrm{PRS}} \times \mathrm{SES}$ interaction no support for this was found $(\beta=-0.02,95 \% \mathrm{CI}=-0.07,0.04$, $t(593)=-0.57, p=.569)$, a result that comports with twin data from Australia (Bates et al., 2016; Grasby et al., 2017).

Hypothesis 3, that offspring attainment would be significantly associated with parental EA3 PRS computed from alleles not transmitted to their offspring, reflecting cultural transmission, was updated using a multilevel model, with offspring EA3 PRS, 
parental non-transmitted EA3 PRS and age and sex as predictors. The effect was, again, replicated with increased magnitude (from $\beta=0.058,95 \% \mathrm{CI}=0.002,0.114$ to $\beta=0.068,95 \% \mathrm{CI}=0.014$, $0.123, t(605)=2.45, p=.014$; see Figure 1, panel B). Supporting hypothesis 4 , which predicts that the non-transmitted effect operates via parental socioeconomic competence, controlling parent SES wiped out the non-transmitted allele effect in the model $(\beta=0.00,95 \% \mathrm{CI}=-0.05,0.06, t(594)=0.17, p=.862)$.

\section{Discussion}

The findings of our initial investigation were replicated. In addition to increased magnitude of associations of offspring educational attainment with offspring EA3 PRS, the effect of parent genotype on a parental competence phenotype supporting child cognitive development was replicated, with higher attainment in offspring associated with non-transmitted elements of the EA3 PRS, and an effect size around one-third the magnitude of children's own EA genetic score. The finding supports the view of EA3 gene polymorphisms as underlying a social competence phenotype of which education is just one component. The results also confirmed that the effects of parenting on attainment appear to mediate one or more of the aspects associated with the SES that parents provide their offspring. The useful foci for follow-up research include identifying specific physical and behavioral traits that are causal for improving offspring attainment. Examples of measurable mediators include wealth and housing (Duncan et al., 2011; Galobardes et al., 2006), household chaos (Matheny et al., 1995), as well as parental responsiveness to offspring needs (Bradley \& Corwyn, 2002). The method can also be extended to other outcomes, including psychiatric traits in which parental genotypes may be operating as an extended phenotype influencing offspring.

Acknowledgments. We are greatly appreciative of the support from the twins and their families, and their willingness to participate in our study. We thank Marlene Grace, Ann Eldridge and Natalie Garden for participant recruitment and data collection at QIMR Berghofer Medical Research Institute. We also thank the Queensland Curriculum and Assessment Authority for the provision of QCST data and statistics. Any analyses of QCST data constitutes our own assessment of the data and is not a representation of the agency. This collaborative research was supported by the Human Frontier Science Program (grant number RG0154/1998B) and grants from the Australian Research Council (A79600334, A79906588, A79801419, DP0212016, DP0664638, DP1093900) and the Netherlands Organisation for Scientific Research (NWO) 016.Veni.198.058. L.C-C. was supported by a QIMR Berghofer fellowship.

\section{References}

Bates, T. C., Hansell, N. K., Martin, N. G., \& Wright, M. J. (2016). When does socioeconomic status (SES) moderate the heritability of IQ? No evidence for $\mathrm{g} \times \mathrm{SES}$ interaction for IQ in a representative sample of 1176 Australian adolescent twin pairs. Intelligence, 56, 10-15.

Bates, T. C., Maher, B. S., Medland, S. E., McAloney, K., Wright, M. J., Hansell, N. K., .. Gillespie, N. A. (2018). The nature of nurture: Using a virtualparent design to test parenting effects on children's educational attainment in genotyped families. Twin Research and Human Genetics, 21, 73-83.
Bradley, R. H., \& Corwyn, R. F. (2002). Socioeconomic status and child development. Annual Review of Psychology, 53, 371-399.

Cordell, H. J., Barratt, B. J., \& Clayton, D. G. (2004). Case/pseudocontrol analysis in genetic association studies: A unified framework for detection of genotype and haplotype associations, gene-gene and gene-environment interactions, and parent-of-origin effects. Genetic Epidemiology, 26, 167-185.

Duncan, G. J., Morris, P. A., \& Rodrigues, C. (2011). Does money really matter? Estimating impacts of family income on young children's achievement with data from random-assignment experiments. Developmental Psychology, 47, 1263-1279.

Galobardes, B., Shaw, M., Lawlor, D. A., Lynch, J. W., \& Davey Smith, G. (2006). Indicators of socioeconomic position (part 1). Journal of Epidemiology and Community Health, 60, 7-12.

Grasby, K. L., Coventry, W. L., Byrne, B., \& Olson, R. K. (2017). Little evidence that socioeconomic status modifies heritability of literacy and numeracy in Australia. Child Development. Advance online publication. doi: $10.1111 /$ cdev. 12920

Howey, R. (2014). PseudoCons. Retrieved from http://www.staff.ncl.ac.uk/ richard.howey/pseudocons/

Koellinger, P. D., \& Harden, K. P. (2018). Using nature to understand nurture. Science, 359, 386-387.

Kong, A., Thorleifsson, G., Frigge, M. L., Vilhjalmsson, B. J., Young, A. I., Thorgeirsson, T. E., ... Stefansson, K. (2018). The nature of nurture: Effects of parental genotypes. Science, 359, 424-428.

Lee, J. J., Wedow, R., Okbay, A., Kong, E., Maghzian, O., Zacher, M., ... Cesarini, D. (2018). Gene discovery and polygenic prediction from a genome-wide association study of educational attainment in 1.1 million individuals. Nature Genetics, 50, 1112-1121.

Matheny, A. P., Jr., Wachs, T. D., Ludwig, J. L., \& Phillips, K. (1995). Bringing order out of chaos: Psychometric characteristics of the confusion, hubbub, and order scale. Journal of Applied Developmental Psychology, 16, 429-444.

McMillan, J., Beavis, A., \& Jones, F. L. (2009). A new socioeconomic index for Australia. Journal of Sociology, 45, 123-149.

Okbay, A., Beauchamp, J. P., Fontana, M. A., Lee, J. J., Pers, T. H., Rietveld, C. A., . . Benjamin, D. J. (2016). Genome-wide association study identifies 74 loci associated with educational attainment. Nature, 533, 539-542.

Pinheiro, J., Bates, D., DebRoy, S., Sarkar, D., \& R Core Team. (2017). nlme: Linear and nonlinear mixed effects models (Version $\mathrm{R}$ package version 3.1-131). Retrieved from https://cran.r-project.org/web/packages/nlme/ citation.html

Purcell, S., Neale, B., Todd-Brown, K., Thomas, L., Ferreira, M. A., Bender, D., ... Sham, P. C. (2007). PLINK: A tool set for whole-genome association and population-based linkage analyses. American Journal of Human Genetics, 81, 559-575.

Queensland Studies Authority. (2003). The 2003 Queensland Core Skills Test retrospective. Brisbane, Australia: Queensland Studies Authority.

Tucker-Drob, E. M., \& Bates, T. C. (2016). Large cross-national differences in gene $\mathrm{x}$ socioeconomic status interaction on intelligence. Psychological Science, 27, 138-149.

Wray, N. R., Lee, S. H., Mehta, D., Vinkhuyzen, A. A., Dudbridge, F., \& Middeldorp, C. M. (2014). Research review: Polygenic methods and their application to psychiatric traits. Journal of Child Psychology and Psychiatry, 55, 1068-1087.

Wright, M. J., \& Martin, N. G. (2004). The Brisbane Adolescent Twin Study: Outline of study methods and research projects. Australian Journal of Psychology, 56, 65-78. 